\title{
Prediction of stress shielding around implant screws induced by three-point and four-point bending
}

\author{
Izzawati Baharom a, ${ }^{*}$, Mohd Afendi Rojan a, Mohd Shukry Abdul Majid a, Nor Alia Md Zain b, Mohd \\ Yazid Bajuri ${ }^{c}$ \\ a Fracture and Damage Mechanics (FDM) SIG, School of Mechatronic Engineering, Universiti Malaysia Perlis (UniMAP), 02600 Arau, Perlis, \\ Malaysia \\ ${ }^{b}$ Institute of Mathematics, Universiti Malaysia Perlis (UniMAP), 02600 Arau, Perlis, Malaysia \\ c UKM Medical Centre, Department of Orthopedics and Traumatology, Universiti Kebangsaan Malaysia, Jalan Yaacob Latif, Bandar Tun Razak, \\ Cheras, Kuala Lumpur, Malaysia
}

* Corresponding author: izzawati.basirom@gmail.com

\section{Article history}

Received 10 Mac 2018

Revised 18 May 2018

Accepted 16 August 2018

Published Online 25 August 2019

\begin{abstract}
Implant screws failure commonly occurs due to the load that constantly generated by the patient's body to the fracture area. Bending load is often encountered in femur bone due to lateral impact which affected the bone and also the implants installed. Consequently, the load will lead to the failure of implants that can cause loosening or tightening of implants. Henceforth, in this manner, it is significant to study the bending behavior of bone implant in femur bone. The aim of this study was to analyze the stress shielding of bone implant on the internal fixator. 3D technique is able to show the overall deformation and stress distribution. The lower the biomechanical compatibility, the lower the STP value obtained. In addition, the variation of elastic modulus (E) of the screws materials, 200GPa (Stainless Steel) and 113.8GPa (Titanium) resulted in the increase of the total stress transferred (STP) between screw and bone interface. In this work, strain energy density (SED) was determined as a good indicator of stress shielding.
\end{abstract}

Keywords: Stress shielding, biocompatibility, strain energy density, bone-implant interaction

\section{INTRODUCTION}

Orthopedic screws are primarily responsible for ensuring the stability of the fracture fixation device. Screw loosening is a major concern in implant failure which mainly associated with the pull-out loading (Ramtani \& He, 2014). Pull-out loading induced by the bending loads is applied on the implant based on the compression load which is related to the biomechanical of daily human activities. It can cause complications such as graft failure, loss of range of motion, nerve injury, bone tunnel confluence, and surgical site infection (Helito et al., 2014). Besides, the high-stress concentration causes the loosening of the screw which can result in implant failure and fracture site infection. Screw loosening studies are well-reported in the literature (Gefen, 2001; Gefen, 2002). However, studies with regards to the bone-screw interactions involving the reduction of stress shielding are remained unclear.

Fracture healing is commonly categorized by the three-phase of responses which are inflammation, repair, and-remodeling phase (Haase \& Rouhi, 2013). By these means, they have acted as references to a better knowledge of bone healing simulation including the conceptual modeling and biomechanical modeling. In bone healing simulation, many researchers merely focus on fixed callus geometry to represent a certain level of bone growth (Sun \& Swain, 2017). Some studies focus on the effect of the treatment outcome and the factors that influence the fracture healing process such as external loading, type and duration of fixation, and the morphology of fracture (Ghiasi et al.,
2017; Shibata et al., 2015). This modeling simulation covers only geometry of initial bone fracture structure but does not include the structural remodeling or directional properties in bone. However, the interface between implant can be varied based on material properties at a certain level of bone remodeling phase.

Furthermore, the healing environment of the fracture site is affected by the stiffness of the fracture site. Fracture stiffness is reflected as a reliable measurement of clinical union and linear elastic properties of callus. Likewise, improvement and issues concerning Osseo-integrated implant have become an interest for worldwide researchers (Gaviria et al., 2014). The nature of implant insertion has been examined for years through pre-clinical models (Stadlinger et al., 2013). The aim is to highlight the factors that promote the success of implantation by examining the prerequisites for bone formation: material, design, and loading condition (Albrektsson, 2008). The early implant integration is improved by systemic treatments in promoting the osteogenesis (Dayer et al., 2007; Smeets et al., 2016). Thus, the analysis involving interaction between bone implant is necessary to investigate the stress concentration on the fracture site while considering the biological, biomechanics, and mechanical behavior knowledge in the model.

The 2D modeling representation of bone implant structure is based on assumption that the load is axially symmetrical (Lin et al., 2007). Most of 2D model studies give enough insight into the behavior of bone implant interface. By comparing to the other studies, it is claimed that the stress analysis of bone predicted by 2D model is less accurate than that provided by 3D model (Cheng et al., 2017; Lin et al., 2007). 
Assumptions of 2D model will only be considered of a cross-section view although it is capable to provide an accurate information in tissue bone around the implant. However, a 3D modeling technique must be adopted first to determine the external loading in order to show the clear overall deformation and stress distribution.

Some researchers were deliberated in bone remodeling theory of 2D axisymmetric configuration which represents the implant, cortical, and trabecular bone region (Vanegas et al., 2011; Chou \& Muftu, 2013). They assumed that the osteotomy gap to be filled with callus tissue is either immature or matured bone. Seeing that the interaction between implant and bone is created while the external load is applied, there are stresses distributed along the implant screw which subsequently transferred to the neighboring bone. A simple model of the screws with the threads is employed as an anchorage in cortical and trabecular bone. The threaded screw functioned as a model to represent the interfacial bone implant contact conditions as shown in Fig. 1.

Additionally, stress shielding can be determined by parametric study in the form of stress transfer parameter (STP) and strain energy density transfer parameters (SEDTP). Ensuing to this, the aim of these approaches is being utilized to calculate the effective stresses at the defined points in the trabecular bone and screw threads (Haase \& Rouhi, 2013). Loosening of bone screws caused by stress shielding and subsequent unbalanced bone remodeling processes have showed the result of bone loss around the screws which could ultimately lead to bone fixation failure. Thus, it is pertinent to investigate the interaction between the orthopedic screw and bone using FEA. Hence, the objective of this study was to analyze the bone-implant fixator stressstrain behavior subjected to three point and four-point bending as this has become a fundamental issue to understand the effect of bending load on the stress-strain behavior of the bone and screws which caused breakage and pain to the outpatient.

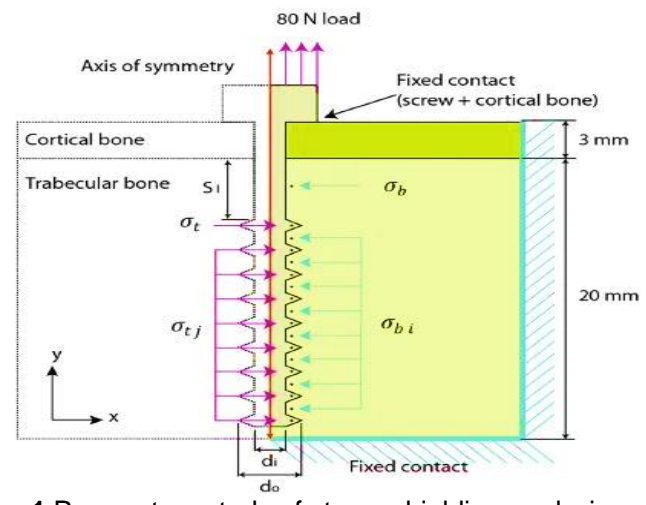

Fig. 1 Parameters study of stress shielding analysis.

(Haase \& Rouhi, 2013)

\section{Finite element modelling}

Fig. 2 shows the flowchart of the stress shielding analysis. CAD Design of standard femur bone was obtained from www.biomedtown.org portal. The geometry of femur bone took the shape created from CT database through the image segmentation with IGES format and the model was further modified by placing the cavity in the femur to represent the trabecular bone section. Next, conventional cortex screw was designed by parameters for bone fracture fixation based on Synthes product. The finite element analysis was developed using ANSYS Workbench 18v and the optimized mesh was via appropriate setting together with values in reaching a small element or number on proximities and curvatures for the model. Convergence test was also performed as reported in the previous study (Izzawati et al., 2017a). Following to this, the number of tetrahedral elements for femur bone and converge number of elements; 1058958, was used for the bone fracture fixation model. Plus, an appropriate setting and values have been executed in order to employ smaller elements on proximities and curvatures on behalf of the model. From this, the simulation results would be more conservative in respect to the converged element numbers in which the accuracy of prediction and adjustment of the governing parameters shall validate the models. Error within tolerance. The maximum von-Mises stress was compared within the tolerance of the yield strength of bone and implant material. Fig. 3 shows the meshed model of four-bending.

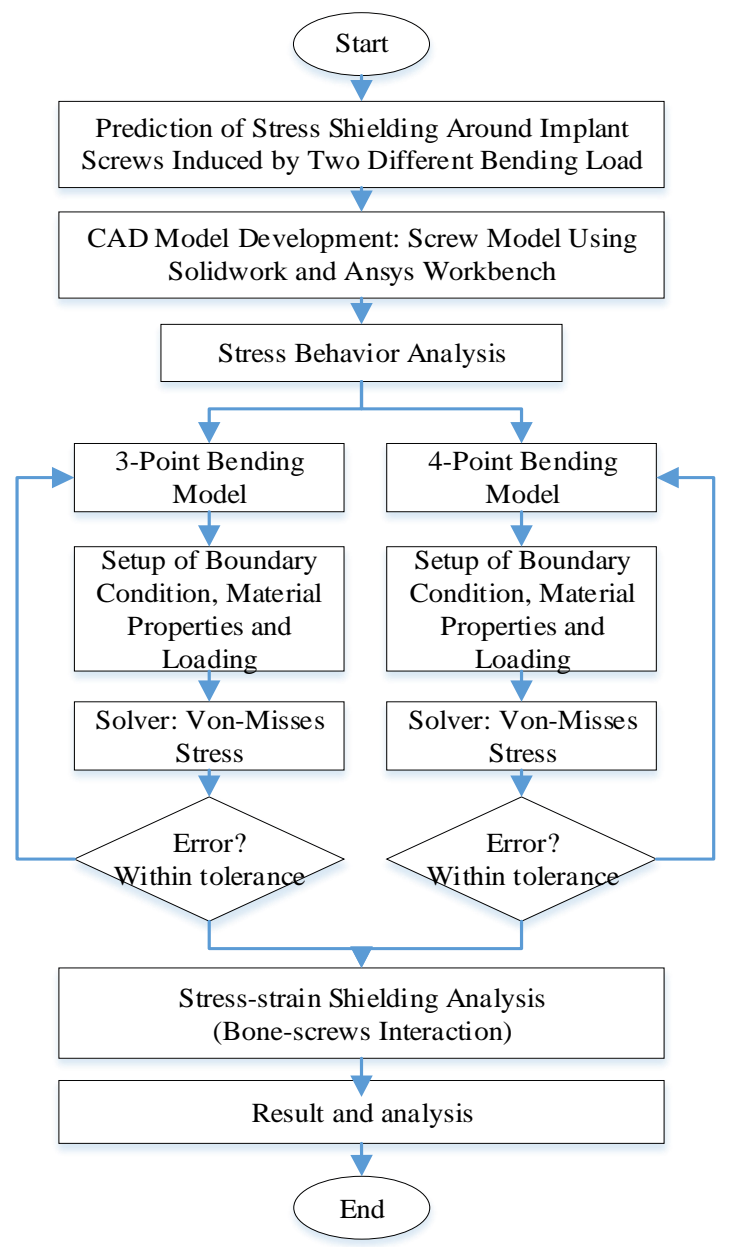

Fig. 2 Flow process.

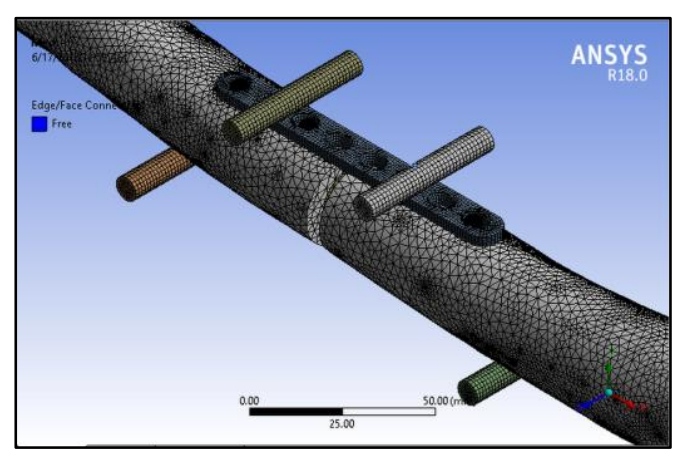

Fig 3 Meshed model.

Table 1 describes the details of bone fracture fixation for conventional cortex screw and compression plate based on Synthes product. In this study, the screw length of (i.e. $36 \mathrm{~mm}$ ), and two types of material (i.e. Stainless Steel and Titanium) were used. These types of bone fracture fixation were chose based upon the fracture type as suggested the AO Foundation (Colton \& Pape, 2017). In addition, the models of bone implant fracture were utilized to concentrate on diaphysis shaft fracture. The implant model selected for the surgical approach was based on the AO Foundation, as a simple fracture. As such, the implant model used conventional cortical screw and straight compression plate. Table 2 represents the information regarding the material properties of bone; cortical bones were assumed to be orthotropic, homogeneous, and linearly elastics, similar as the other materials used in this study.

Furthermore, the bone fracture fixation model consisted of a compression plate and four internal conventional cortex screws 
(full threaded) being anchored along the diaphysis shaft as shown in Fig. 4 (a). The fixation or implant was placed in the middle of the fracture line. Also, the fracture gap was created approximately $5 \mathrm{~mm}$ and the screws orientation was fixed at $90^{\circ}$ based on the fracture angle. The stability of the fracture surfaces is important to determine the precondition of successful healing. Fracture gap was required to allow the formation of new bone tissue as it is capable to simulate the fracture on the diaphysis shaft. In addition, screw orientation of $90^{\circ}$ was selected to examine the simple fracture of $0^{\circ}$. Although the previous studies showed that $30^{\circ}$ was a critical stress concentration on implant screws (Izzawati et al., 2017), the results showed that $0^{\circ}$ fracture angle has $20 \%$ less than the stress based of $30^{\circ}$ fracture angle. As for the stress shielding, the bone-implant interface was also considered. Thus, this study was aimed to obtain the flexural strength of implant and stress shielding while neglecting the screws orientation. Both parts of model were imported into Ansys Workbench $18 \mathrm{~V}$ software and assembled by Boolean operation in design modular. Fig 4(b) shows the crosssectional view along the femur bone model. Each screw implanted through the bone (i.e. pass through the average of $4.4 \mathrm{~mm}$ thickness of cortical bone and cavity filled with trabecular bone).

Table 1 Dimensional parameters for bone fracture fixation.

\begin{tabular}{lcll}
\hline $\begin{array}{c}\text { Conventional Cortex Screw } \\
(\mathbf{m m})\end{array}$ & \multicolumn{1}{c}{$\begin{array}{c}\text { 8-holes compression plate } \\
(\mathbf{m m})\end{array}$} \\
\hline Diameter of thread, $d_{o}$ & 3.5 & Width & 11.0 \\
Thread pitch & 1.25 & Thickness & 3.3 \\
Diameter of core, $d_{i}$ & 2.4 & Center-to-hole distance & 13.0 \\
Diameter of head & 6.0 & & \\
\hline
\end{tabular}

Table 2 Mechanical properties of bone structure and implant materials.

\begin{tabular}{llll}
\hline Bone material & \multicolumn{1}{c}{$\begin{array}{c}\text { Young's } \\
\text { modulus,E } \\
(\mathrm{GPa})\end{array}$} & $\begin{array}{c}\text { Poisson's } \\
\text { ratio }(\mathbf{v})\end{array}$ & $\begin{array}{c}\text { Shear } \\
\text { modulus } \\
(\mathrm{GPa})\end{array}$ \\
\hline Trabecular & $\mathrm{E}_{\mathrm{Tb}}=1.1$ & $\mathrm{U}_{\mathrm{Tb}}=0.3$ & - \\
$\begin{array}{l}\text { Cortical Bone } \\
\text { (Longitudinal }\end{array}$ & $\mathrm{E}_{3}=20.0$ & $\mathrm{U}_{12}=0.376$ & $\mathrm{G}_{12}=4.53$ \\
transverse) & $\mathrm{E}_{1}=12.0$ & $\mathrm{U}_{23}=0.235$ & $\mathrm{G}_{23}=4.53$ \\
& $\mathrm{E}_{2}=12.0$ & $\mathrm{U}_{23}=0.376$ & $\mathrm{G}_{13}=4.53$ \\
Stainless Steel & $\mathrm{E}_{\mathrm{S} . \mathrm{S}}=200$ & $\mathrm{U}_{\mathrm{S} . \mathrm{S}}=0.3$ & - \\
Titanium & $\mathrm{E}_{\mathrm{Ti}}=113.8$ & $\mathrm{U}_{\mathrm{Ti}}=0.34$ & - \\
\hline
\end{tabular}

Two types of analysis were conducted, namely three-point bending and four-point bending. Three-point bending simulation was carried out to obtain the flexural strength of implant in fracture bone. Besides, fourpoint bending was performed to obtain the strength of bone and implant, in which, this approach is proposed for biomechanical test of bone due to the orthotropic behavior of bone. This type of bending is often found in femur bone due to lateral and compressive impact such as walkers and vehicle passengers from accidents. Femur bone may be considered as having two main functions which are support and structures on which muscles may act to generate motion. Both functions require it to be stiff and deform where the bending load applied due to body weight and joint reaction force or abductor force about $10 \mathrm{~mm}$ in coronal plane. The existence of a fracture gap generates the pressure load distribute from the proximal portion to the distal part through the implant. Afterwards, stress analyses were performed to obtain Vonmisses stress for bone and screws. However, it was done to a certain extend only to study the stress shielding in 3D. Thus, cross sectional geometry was also made along the diaphysis shaft and implants. The stress probe was used to callout the stress data on each of screws thread and neighboring bone too. The use of this technique is to obtain the stress transfer at certain location in bone and implant in the same way of 2D approach. Similar finding has proposed that 3D model approach can be used in order to obtain a better understanding of bone behavior in term of biomechanical perspective (Idhammad et al., 2013). A challenging assumption is often risky, since it carries a question mark to the existing knowledge that the researcher will be disqualified of bone healing (Isaksson, Donkelaar, \& Ito, 2009), but also to the mechanical implication of certain loading conditions, fixation device, or stress strain energy released in the fracture callus (Shefelbine et al., 2005). Thus, the consideration of 3D and 2D model techniques as well as studies of biomechanical and interaction of bone-implant are helpful for further investigation.
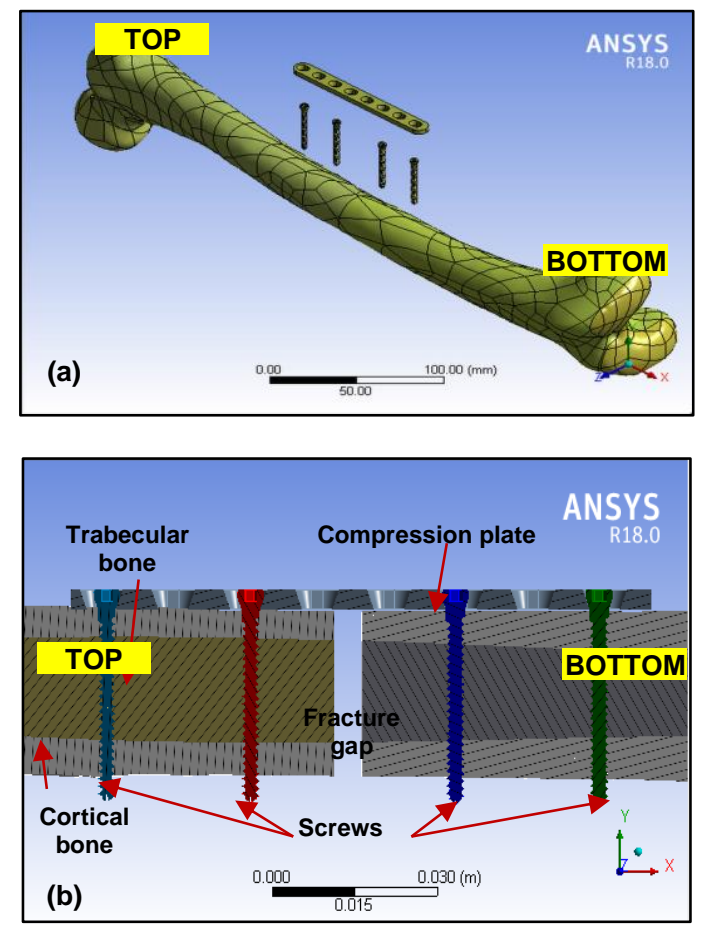

Fig. 4 (a) Bone fracture fixation model (b) Cross sectional view of bone fracture fixation model.
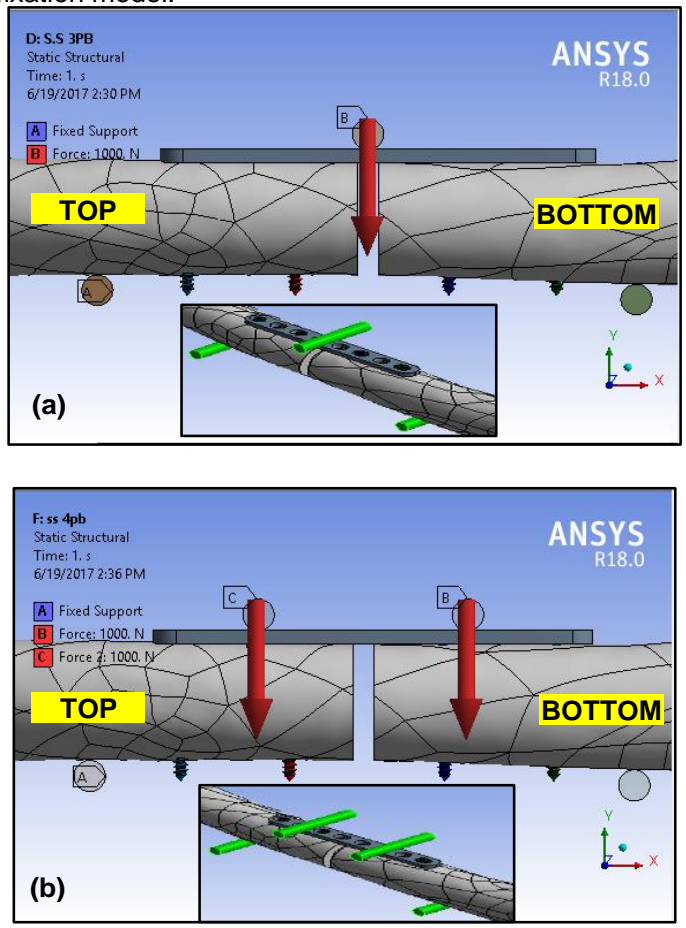

Fig. 5 Boundary condition of (a) three-point and (b) four-point bending.

There are two types of bending being applied to the models which are three-point and four-point bending as shown in Fig. 5. Plus, there were also pins of fixator for bending simulation. Fig. 5(a) shows Pin A and Pin $C$ as the fixation point while Pin B as the loading point crossed to the implant for bending simulation. Fig. 5 (b) shows four- point bending model wherein Pin A and Pin D were setup up as fixation point whilst 
Pin B and Pin C as loading point. A uniaxial compression load of 1000 $\mathrm{N}$ along y-component was applied on the upper cylindrical beam where it has been placed directly on the implant crossing between the fracture gap. The model was further supported by two cylindrical beams below the bone model with the gap of $186 \mathrm{~mm}$. Subsequently, the results of von-Mises stress were investigated to determine the critical screw failure.

Furthermore, the interactions between bone and screw were analyzed by using stress shielding approach in which the twodimensional of cross section view was deployed. Shielding analysis emphasized on the critical screw while the stress in a proximal thread and distal thread on screw and bone were determined. In order to classify these stresses, stress transfer parameter (STP) and strain energy density transfer parameter (SEDTP) were calculated by implementing the effective stresses at the defined points in the trabecular bone and screv $_{\alpha=\sigma_{b}}^{\text {thrond }} / \sigma_{t}^{\text {Stress transfer parameters are defined as }}$

$$
\begin{array}{lcc} 
& \\
\operatorname{STP} & =\sum_{i=j=2}^{i=j=N}\left(\sigma_{b i} / \sigma_{t j}\right) \\
\operatorname{STP} & \alpha & \beta
\end{array}
$$

STP total $=$ STP + STP

and strain energy density transfer parameter (SEDTP) are expressed

$$
\begin{aligned}
& \text { as } \quad \begin{array}{l}
\alpha=\sigma_{b} \varepsilon_{b} / \sigma_{t} \varepsilon_{t} \\
\beta=\sum_{i=j=2}^{i=j=N}\left(\sigma_{b i} \varepsilon_{b i} / \sigma_{t j} \varepsilon_{t j}\right) \\
\text { SEDTP }
\end{array} \quad \alpha \quad \beta \\
& \text { SEDTP }
\end{aligned}
$$$$
\text { SEDTP total }=\text { SEDTP }+ \text { SEDTP }
$$

where STP $\alpha$ and STP $\beta$ are the ratios of equivalent stress transferred to the bone $\left(\sigma_{b}\right)$ and neighboring screw thread $\left(\sigma_{t}\right)$. SEDTP are defined to evaluate the stress transfer involving stress-strain behavior of the model, von-Mises stress $(\sigma)$ and von Mises strain $(\varepsilon)$. The subscripts $b$ and $t$ are referring the bone and threads, respectively.

\section{RESULTS AND DISCUSSION}

Table 3 represents the tabulated data of maximum von-Mises stress of screw for both materials and bending loads. The results showed the Stainless Steel had a higher stress in comparison to the Titanium for both bending loads. The reason for this is due to the Stainless Steel that had higher elastic modulus compared to Titanium which consequently led the Stainless Steel to the optimum failure strength. The modulus obtained by three-point and four-point bending was different, but it has the $1 \%$ value which corresponds to the stress-strain slope. Although there were different moduli between these loads, but the small values did correspond to the same method. The critical screw stress was investigated after the bending load applied. Critical stresses were focused on the bone and screws model while the compression plate was ignored. This is for the presence of compression plate which acted as the real case of implant devices used in bone-implant fixation.

In addition, Fig. 6 shows the total deformation of bone implant fixation under bending load at $5 \mathrm{~mm}$ fracture gap. This model is not considered as the bone remodeling, since the fracture gap was not filled with callus or soft bone tissue. This was done to obtain the actual flexural strength of implant without attachment of soft bone tissue. Following to this, the critical stress of flexural strength was obtained for implant on three-point bending simulation. As what has been found in the deformation, compression plate and screws displayed a significant effect as loads applied. The maximum stress was created by bending pins to the compression plate directly transferred to the screws. Appropriately, to obtain the critical stress for stress shielding, the vonMises stresses of screws and bone were obtained. Critical stress was put into priority on the screw neck. Thus, the stress shielding evaluation was conducted on the critical stress of concentrated screw.
Table 3 Maximum von-Mises stress of stainless steel and titanium screws at two types of bending load.

\begin{tabular}{llc}
\hline Bending type & \multicolumn{1}{c}{ Materials } & $\begin{array}{c}\text { von-Mises stress } \\
\text { (MPa) }\end{array}$ \\
\hline 3-point & Stainless steel (S.S) & 160 \\
bending & Titanium (Ti) & 125.44 \\
\hline 4-point & Stainless steel (S.S) & 183.08 \\
bending & Titanium (Ti) & 162.63 \\
\hline
\end{tabular}

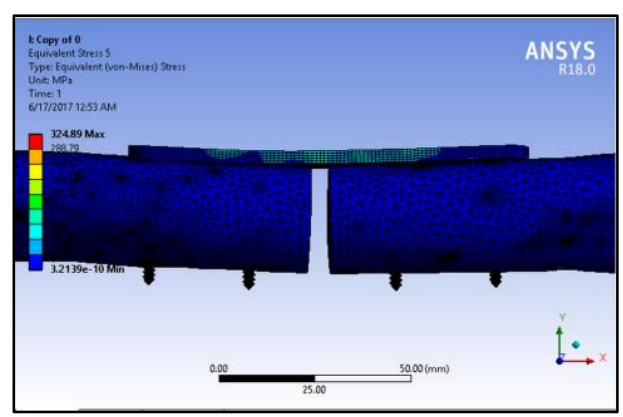

Fig. 6 Total Deformation of bone-implant.

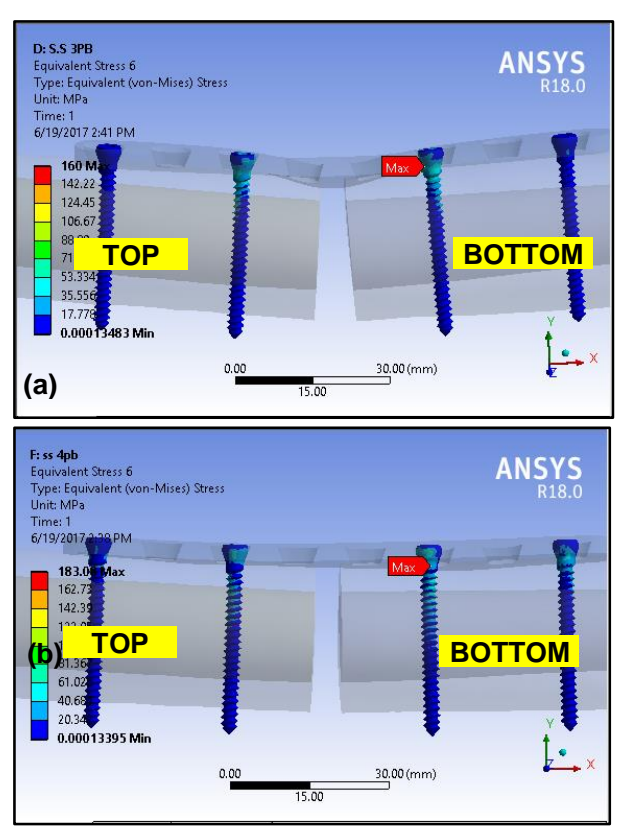

Fig. 7 Maximum von-Mises stress found in the screws near the fracture site.

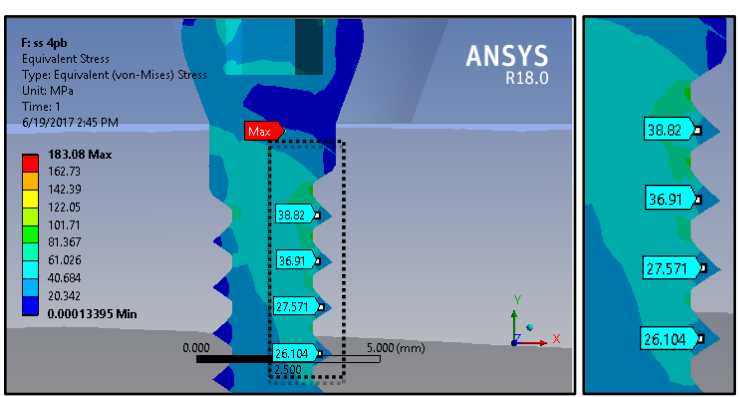

Fig. 8 Enlargement of stress contour of the screw as defined by parameters of STP and SEDTP.

Accordingly, Fig. 7(a) and Fig. 7(b) exhibit the results of the 3-point and 4-point bending, respectively. It can be clearly seen that critical stress was present on the screw near the fracture site for both bending loads. Afterwards, the stress distributions in bone and screw 
from the bending load were determined using two-dimensional of the cross section view along the diaphysis shaft. By deploying the defined parameters in Eqs (1)-(6), STPs and SEDTPs stress values were determined on the screw shaft and the neighboring bone as shown in Fig. 7.

Furthermore, the result displays the evaluation and rating of biomechanical compatibilities for bone-implant screws materials. The results show maximum von-Mises stress existed in the intersection of the screw head and the profile. On that account, the stress shows reduction at the lowering readings to the screw tip, which the average stress of neighboring screw thread $\left(\sigma_{t}\right)$ to trabecular bone volume is located directly above it and withstand the average of the bone $\left(\sigma_{b}\right)$. The first of proximal thread has higher distal thread (following thread) since the bone was compressed between first thread of the implant screws and head of screw as the first thread carried a substantial load, this gave result of the stress concentration to appear above it. This is also the reason of stress transfer analysis being performed separately between the first and following thread. Based on the stress concentration contour of neighbor bone, the prediction of bone resorption could be predicted. The highest stress concentration shows less bone resorption takes place around the screw neck. The prediction can be proven by the images reported by Schuller-Gotzburg et al. that illustrated the most of bone resorption occured around the screw's tip while resorption on head and shaft of screws were distinctly minimal (Stahel et al., 2017; White et al., 2016)

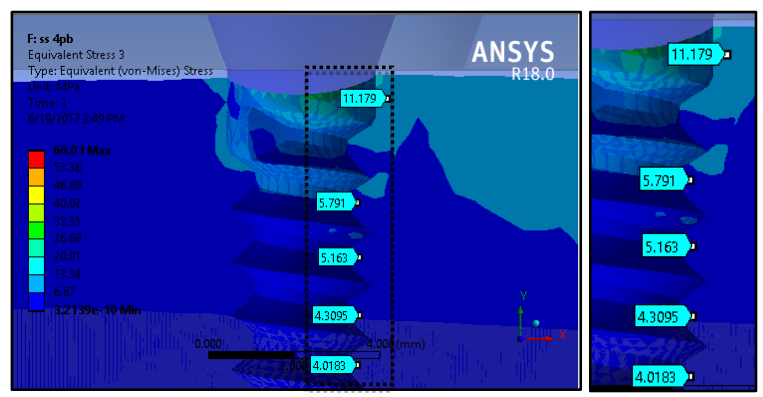

Fig. 9 Enlargement of stress contour of the neighboring bone.

This is discovered to be similar to the von-Mises strain obtained in the screw thread which has a critical strain on the first thread. Nonetheless, maximum von-Misses were determined, in order to obtain the stress being transferred to the neighboring bone. Fig. 9 shows the result of maximum von-Mises stress obtained in the bone of the neck interfaces. The stress presents reduction along the screws profile which correlated to the von-Mises strain obtained in the bone thread that has a critical strain on the first thread. This critical stress found in the screw neck was expected to have the largest in vitro shear stress (Vijayalakshmi et al., 2012; Huang et al., 2015). The statement was also further supported by the stress contour results that showing the thread being closest to the cortical bone region has higher stresses concentration.

Besides, the stresses were measured on the both proximal thread $(\alpha)$ and distal thread $(\beta)$ to achieve the stress transfer parameters. The obtained results show significant and greater stress transferred on STP $\alpha$ compared to the STP $\beta$ for both loads as shown in Fig. 7(a). Moreover, STP total of Stainless Steel screws shows less stress transfer to the bone compared to the Titanium for the two bending loads. This is due to the reason that the stainless steel has higher elastic modulus in contrast to the titanium for both bending loads. It is learned that the titanium screws have a good mechanical stimulus, in term of stress transfer which is expected to attain the high level of stability in promoting the bone remodeling process. In addition, as a matter of fact, SED transfer parameter is also very pertinent for the initiation and bone remodeling process.

As for the stress transfer parameters evaluation, the implant screw was made of material properties with types of property that are equivalent to those of their host or bone of which the screw and bone will share the similar loads and a nearly homogeneous stress transfer result. The ideal value of STP is $0.96-0.99$ for both STP on proximal thread $(\alpha)$ and distal thread ( $(6)$. The hypothesis of biocompatibility and stress transfer parameter was made wherein; the lower biomechanical compatibility (e.g. materials are significantly stiffer than bone), the lower STP value is obtained. Based on the result in Figure 10 (a), the stainless steel shows a lower biomechanical compatibility compared to the titanium, where STP total value (S.S) was less than the STP total value (Ti). The two materials show at the first thread of screw whilst the neighboring bone shows a high stress transfer compared to subsequent thread.
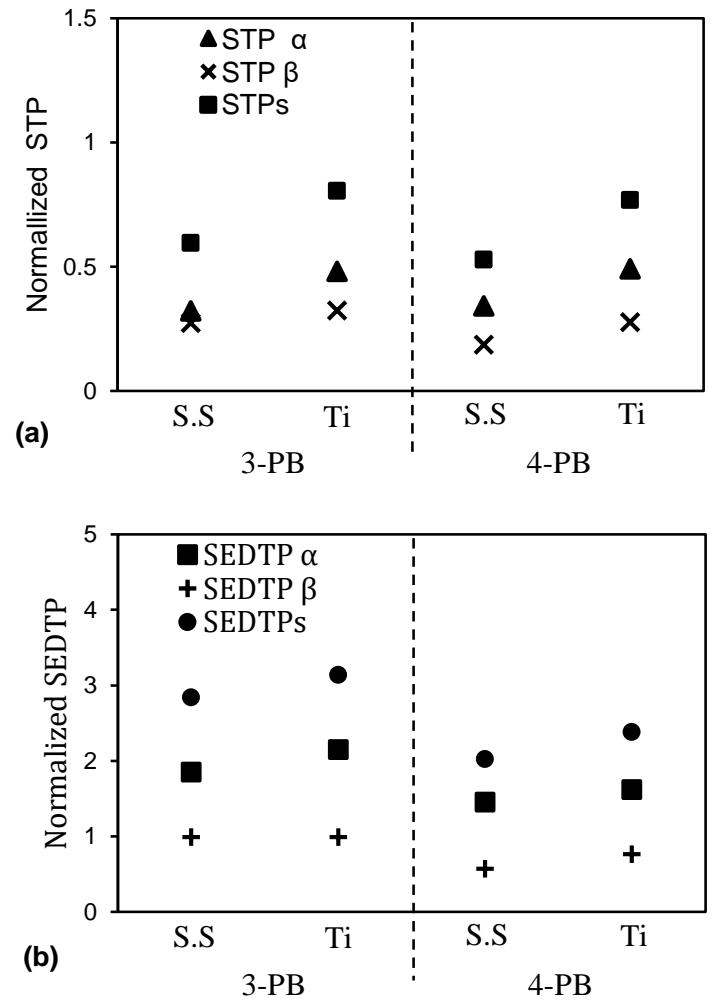

Fig. 10 The effect of screws' material on average STPS/ SEDTPS (a) three-point (3-PB) and (b) four-point bending load (4-PB).

To simplify this, the overall average result of STPs and SEDTPs under the there-point bending is higher than the four-point bending. This might be the contact zone between implant model and cylindrical supports. Further, the total deflections involve the axial forces along the beam which caused the different level flexural strength since the bending moment deformed has distributed stresses on the screw thread and bone interfaces. Thus, there were significant differences in the first thread and distal thread based on the STPs and SEDTPs criterion as shown in Fig. 10 (b).

SEDTP $\beta$ under three-point bending shows a linear decrease in correlation with STP $\beta$, compared to the SEDTP $\alpha$ which shows a highly reaction to the SEDTPs values in both loads. These results are achieved due to the existence of adjacent force or pull out loading alongside the screw head which subsequently distributed the strain energy throughout the screw shaft. The variation of elastic modulus (E) of the screws' materials; $200 \mathrm{GPa}$ (stainless steel) and $113.8 \mathrm{GPa}$ (titanium) have resulted in increasing the total stress transferred, STPs between screw and bone interface. The increment on average STPs was of $26 \%$ (stainless steel) and $31 \%$ (titanium) for three-point and fourpoint bending load respectively. It is also observed that both types of load show an escalation on the average of STPs for the simulated titanium screws, in comparison to the stainless-steel screws as shown in Fig. 11(a) and Fig. 11(b). This result denotes an agreement with the literature finding wherein it is stated that the increase of elastic modulus of the screws causes the increase of stress shielding (Gefen, 2001).

Intensifying the screw's elastic modulus certainly had a significant effect on the average SEDTPs. As examined, the increament is about $10 \%$ and $15 \%$ of the average of SEDTPs for the three and four-point bending loads respectively. These results were contrary to the previous 
studies which stated that the elastic modulus had no effect on average (SEDTPs) (Haase \& Rouhi, 2013). Nevertheless, the obtained results were dependent on the variability of geometric parameters and materials properties.

What is more, the load transfer between screw and neighboring bone is actually better than what has been predicted using 2D model. The stress shielding evaluation is not limited to the forces along the implant screw, but it also involved bending and shear as the additional stress bounded between the screw thread should be considered. Hence, further investigation is needed to provide the bone remodeling, Osseo integration and screw bone interaction contact.
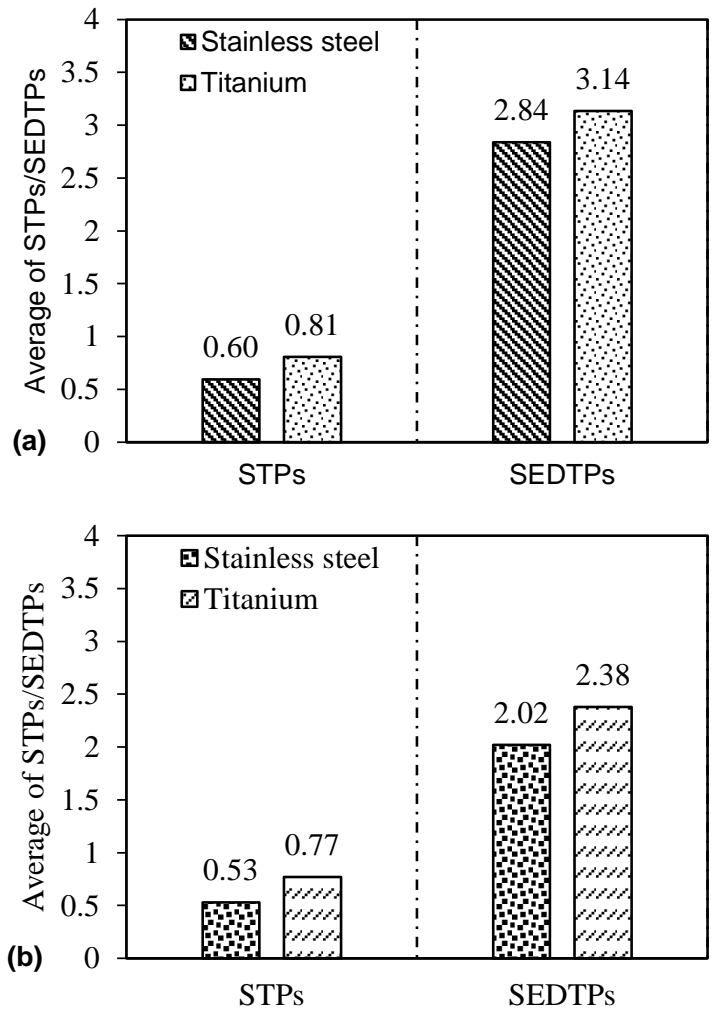

Fig. 10 The effect of screws' material on average STPS/ SEDTPs (a) 3-point bending load (3-PB) and (b) 4-point bending load (4-PB).

\section{CONCLUSION}

To put this into deduction, the stainless steel has higher stress shielding in contrast to the titanium under three-point and four-point bending. The increment on average STPs was of $26 \%$ (stainless steel) and $31 \%$ (titanium) for three-point and four-point bending load respectively. SEDTPs for the three and four-point bending increase about $10 \%$ and $15 \%$ of the average of loads respectively. The maximum flexural strength of implant can be attained when the fracture gap is neglected. Four-point bending shows the intact bone strength for the mechanical behavior of orthotropic elastic material has a significant effect on bone strength. It is due to the directional load that was being applied along the implant which induced the directional stress to be formed.

Additionally, the result is obtained based on the stress transfer STPs $(\alpha$ and $\beta)$ and strain energy density transfer SEDTPs $(\alpha$ and $\beta)$ parameters. The ideal value of STP is $0.96-0.99$ for both STP on proximal thread $(\alpha)$ and distal thread $(\beta)$. Titanium material shows high biocompatibility compare to stainless steel. It is revealed based on 0.81 and 0.77 of STP and SEDTP values, respectively. The screw which was located at the closest of the fracture site has the highest stress concentrated on the screw neck. This study has provided meaningful differences between the stress and strain energy density in the bone under two types of bending loading, which can ultimately assist the bone remodeling rate as well as the stress shielding for different materials.
Plus, this study has set aside the callus formation surrounding the implant screws and between the fracture gap. Despite the present of callus has significant effect on the stress transfer, the homogenous model being used to virtualize the boundary condition of bending has also become necessary to take into consideration.

FEA is reliable to give thought wherein the $2 \mathrm{D}$ model provides accurate information for biocompatibility whilst the 3D technique able to adapt the actual external loading in showing the clear overall deformation and stress distribution. The resistance of the bone against damage by repairing itself and adapting to environmental conditions is the most important property and henceforth, there is a need for a further study. These adaptive changes are regulated by physiological process which commonly called as the bone remodeling.

\section{ACKNOWLEDGEMENT}

This work was financially supported by the Ministry of Higher Education (MOHE) Malaysia and Universiti Malaysia Perlis through the Fundamental Research Grant Scheme FRGS/1/2016/TK03/UNIMAP/03/4)- (FRGS 9003-00578).

\section{REFERENCES}

Albrektsson, T. (2008). Hard tissue implant interface. Australian Dental Journal, 53, 34-38.

Cheng, H., Peng, B., Chen, M., Huang, C., Lin, Y., Shen, Y. (2017). Influence of deformation and stress between bone and implant from various bite forces by numerical simulation analysis, 2017.

Chou, H.-Y., Muftu, S. (2013). Simulation of pen-implant bone healing due to immediate loading in dental implant treatments. Journal of Biomechanics, 46, 871-878.

Dayer, R., Badoud, I., Ammann, P. (2007). Defective Implant osseointegration under protein undernutrition: Prevention by PTH or Pamidronate. Journal of Bone and Mineral Research, 22(10), $1526-1533$.

Gaviria, L., Salcido, J. P., Guda, T., Ong, J. L. (2014). Current trends in dental implants. Journal of the Korean Association of Oral and Maxillofacial Surgeons, 40(2), 50-60.

Gefen, A. (2002). Computational simulations of stress shielding and bone resorption around existing and computer-designed orthopaedic screws. Medical \& Biological Engineering \& Computing, 40(3), 311-322.

Gefen, A. (2001). Dynamic simulations of cancellous bone resorption around orthopaedic fixative implants. International Conference of the IEEE Engineering in Medicine and Biology Society, 23-26.

Ghiasi, M. S., Chen, J., Vaziri, A., Rodriguez, E. K., Nazarian, A. (2017). Bone fracture healing in mechanobiological modeling: A review of principles and methods. Bone Reports, 6, 87-100.

Haase, K., Rouhi, G. (2013). Prediction of stress shielding around an orthopedic screw: Using stress and strain energy density as mechanical stimuli. Computers in Biology and Medicine, 43(11), 17481757.

Helito, C. P., Bonadio, M. B., Demange, M. K., Albuquerque, R. F. da M. e, P??cora, J. R., Camanho, G. L., \& Angelini, F. J. (2014). Screw loosening and iliotibial band friction after posterolateral corner reconstruction. Knee, 21(3), 769-773.

Huang, X., Zhi, Z., Yu, B., Chen, F. (2015). Stress and stability of platescrew fixation and screw fixation in the treatment of Schatzker type IV medial tibial plateau fracture: A comparative finite element study. Journal of Orthopaedic Surgery and Research, 10(1), 1-9.

Idhammad, A., Abdali, A., Alaa, N. (2013). Computational simulation of the bone remodeling using the finite element method: an elasticdamage theory for small displacements. Theoretical Biology and Medical Modelling, 10(1), 32, 1-11.

Isaksson, H., Donkelaar, C. C. Van, Ito, K. (2009). Sensitivity of tissue differentiation and bone healing predictions to tissue properties, 42 , $555-564$.

Izzawati, B., Daud, R., Afendi, M., Majid, M. S. A., Zain, N. A. M. (2017a). Convergence and stress analysis of the homogeneous structure of human femur bone during standing up condition. In AIP Conference Proceedings (Vol. 1885). 
Izzawati, B., Daud, R., Afendi, M., Majid, M. S. A., Zain, N. A. M. (2017b). Stress analysis of implant-bone fixation at different fracture angle Stress analysis of implant-bone fixation at different fracture angle. In Journal of Physics: Conference Series.

Lin, D., Li, Q., Li, W., Ichim, I., Swain, M. (2007). Evaluation of dental implant induced bone remodelling by using 2D Finite element. Biomaterials, (December).

Ramtani, S., He, Q. C. (2014). Internal bone remodeling induced by metallic pin fitted into medulla of a long bone having cylindrical anisotropy: Theoretical predictions. International Journal of Engineering Science, 82, 124-139.

Shefelbine, S. J., Simon, U., Claes, L., Gold, A., Gabet, Y., Bab, I., ... Augat, P. (2005). Prediction of fracture callus mechanical properties using micro-CT images and voxel-based finite element analysis, 36, 480-488.

Shibata, Y., Tanimoto, Y., Maruyama, N., Nagakura, M. (2015). A review of improved fixation methods for dental implants. Part II: Biomechanical integrity at bone-implant interface. Journal of Prosthodontic Research, 59(2), 84-95.

Smeets, R., Stadlinger, B., Schwarz, F., Beck-broichsitter, B., Jung, O., Precht, C., ... Ebker, T. (2016). Impact of dental implant surface modifications on osseointegration. BioMed Research International, 2016.

Stadlinger, B., Korn, P., Eckelt, U., Range, U., Ferguson, S. J., Kramer, I., ... Area, M. D. (2013). Osseointegration of biochemically modified implants in an osteoporosis rodent model. European Cells and Materials, 25, 326-340.

Stahel, P. F., Alfonso, N. A., Henderson, C., Baldini, T. (2017). Introducing the "Bone-Screw-Fastener" for improved screw fixation in orthopedic surgery: a revolutionary paradigm shift? Patient Safety in Surgery, 11(1), 6.

Sun, G., Swain, M. V. (2017). Surface morphology optimization for osseointegration of coated implants. Biomaterials, 31(27), 71967204.

Vanegas-Acosta, J. C., Landinez P., N. S., Garzón-Alvarado, D. A., Casale R., M. C. (2011). A finite element method approach for the mechanobiological modeling of the osseointegration of a dental implant. Computer Methods and Programs in Biomedicine, 101(3), 297-314.

Vijayalakshmi, P. S., Veereshi, A., Jayade, V. P., Dinesh, M., Kumar, M. (2012). Finite Element analysis of stress and strain distribution in the bone around the. The Journal Indian Orthodontic Society, 46(December), 175-182.

White, B. J., Hawkes, T. K., \& Herzog, M. M. (2016). Hip Pain After a Femoral Fracture: It Is Not Always Related to the Implant. Orthopedics, 1-5. 